\title{
Perturbative and non-perturbative aspects of moments of the thrust distribution in $e^{+} e^{-}$ annihilation*
}

\author{
Einan Gardi \\ Laboratoire de Physique Théorique $\dagger$ Université de Paris XI, \\ 91405 Orsay Cedex, France \\ e-mail: Einan.Gardi@th.u-psud.fr
}

\begin{abstract}
Resummation and power-corrections play a crucial role in the phenomenology of event-shape variables like the thrust $T$. Previous investigations showed that the perturbative contribution to the average thrust is dominated by gluons of small invariant mass, of the order of $10 \%$ of $Q$, where $Q$ is the center-of-mass energy. The effect of soft gluons is also important, leading to a non-perturbative $1 / Q$ correction. These conclusions are based on renormalon analysis in the single dressed gluon (SDG) approximation. Here we analyze higher moments of the thrust distribution using a similar technique. We find that the characteristic gluon invariant mass contributing to $\left\langle(1-T)^{m}>\right.$ increases with $m$. Yet, for $m=2$ this scale is quite low, around $27 \%$ of $Q$, and therefore renormalon resummation is still very important. On the other hand, the power-correction to $\left.<(1-T)^{2}\right\rangle$ from a single soft gluon emission is found to be highly suppressed: it scales as $1 / Q^{3}$. In practice, $<(1-T)^{2}>$ and higher moments depend also on soft gluon emission from configurations of three hard partons, which may lead to $\alpha_{s}\left(Q^{2}\right) / Q$ power-corrections. This issue is yet to be investigated.
\end{abstract}

KEYWORDs: QCD, Renormalization Regularization and Renormalons, Jets.

\footnotetext{
*Research supported by the EC program "Training and Mobility of Researchers", Network "QCD and Particle Structure", contract ERBFMRXCT980194.

${ }^{\dagger}$ CNRS UMR 8627
} 


\section{Contents}

1. Introduction 1

2. The thrust distribution in the single dressed gluon model 4

3. Moments of $1-$ thrust 8

4. Conclusions 19

A. Asymptotic expansions for the characteristic functions 24

\section{Introduction}

The systematic analysis of event-shape variables in $e^{+} e^{-}$annihilation has become an active research field in the recent years [1]. High quality experimental data in a wide range of center-of-mass energies $Q$ (from 10 up to $200 \mathrm{GeV}$ ) provide an opportunity for precision test of the theory. On the theoretical side, the simple set-up of $e^{+} e^{-}$ annihilation allows to probe directly the QCD vacuum and learn about the interface between perturbative and non-perturbative physics.

Empirically, it is known for quite some time [2] that perturbative approximations to moments of event-shape variables are consistent with experimental data only upon including additive power-corrections. A classical example is the case of the thrust, defined by

$$
T=\frac{\sum_{i}\left|\vec{p}_{i} \cdot \vec{n}_{T}\right|}{\sum_{i}\left|\vec{p}_{i}\right|}
$$

where the summation is over all the final-state particles $\vec{p}_{i}$. The thrust axis $\vec{n}_{T}$ is a unit vector which is set, for a given event, such that $T$ is maximized. Below we shall use the variable $t \equiv 1-T$ which vanishes in the two-jet limit.

For the average thrust, as for several other average event-shape variables, one finds $1 / Q$ corrections, $\left\langle t>\simeq<t>_{\mathrm{PT}}+\lambda / Q\right.$, where $\lambda$ is some hadronic scale. These corrections are quite large at LEP energies. The case of average event-shape variables was intensively studied both theoretically [3]-10] and experimentally.

Here we investigate perturbative and non-perturbative aspects of higher moments of 1 - thrust, $\left\langle t^{m}>\right.$. This subject was addressed before (see e.g. [11, 12, 13, 14]), but it did not receive yet the appropriate attention. Whereas some experimental results are already available [15, 16], many more can be extracted from stored LEP 
data [17]. This analysis is worthwhile: alongside interesting theoretical insights, the investigation of higher moments of event-shape variables may lead to additional precision measurements of $\alpha_{s}$.

Existence of a well-defined perturbative approximation to event-shape variables is guaranteed since they are infrared and collinear safe. However, the remaining sensitivity of these observables to soft and collinear emission is usually quite high and it shows up in the form of large perturbative coefficients. For event-shape distributions close to the two-jet limit (the Sudakov region) the leading logarithms in the perturbative coefficients can be resummed to all-orders [18. Out of this special part of phasespace, and in particular when moments of event-shape variables are considered, only the leading and next-to-leading perturbative terms are known [19, 20, 21]. Since the apparent convergence of these perturbative expansions in a standard renormalizationscheme and scale is slow, resummation seems necessary. A relevant source of large coefficients are renormalon diagrams [6, 7, 9, 14, 22, 23] which reflect the effect of the running-coupling.

One can imagine [7, 24], in analogy with the skeleton expansion in the Abelian theory, a possibility of reorganizing the perturbative expansion such that all diagrams which correspond to dressing a single gluon are summed first, then diagrams with two dressed gluons, etc. Formally, a systematic expansion of this type has not yet been shown to exist. However, the spirit of the Brodsky-Lepage-Mackenzie (BLM) scale setting method [25], one can attempt to identify and resum the single dressed gluon (SDG) terms to all orders. This is the basis of the renormalon resummation methodology, which was applied in many different QCD examples [14]. In the context of event-shape variables in $e^{+} e^{-}$annihilation we mention the case of the longitudinal cross-section [22] and that of the average thrust [7].

Whatever resummation procedure is applied, it is clear that perturbation theory alone cannot predict the observed values of event-shape variables: the perturbative calculation uses quark and gluon fields while the measurement is of hadrons. The effect of hadronization on the observables can be modeled by Monte-Carlo simulations. However, in order to gain some understanding on the nature of confinement in QCD it is favorable to analyze raw hadronic data, and parameterize non-perturbative effects in the simplest possible way.

It was noticed that hadronization does not involve large momentum transfer. Therefore perturbative results calculated in terms of partons can almost be directly compared with the data [1]. Due to the sensitivity of the observables to soft physics some modification of the perturbative result is still necessary. At the perturbative level, infrared sensitivity shows up in the form of infrared renormalons [14]: the coefficients increase fast, asymptotically as $n$ !, and have a constant sign pattern. Consequently, the series is non-summable. Different resummation prescriptions, or "regularizations" of the perturbative sum, differ by power-suppressed terms. This ambiguity must be compensated at the non-perturbative level. In the SDG ap- 
proximation, one can calculate the perturbative sum and extract the form of its ambiguity. This way [6, 9, 23] a perturbative calculation can be used to identify the parametric dependence of non-perturbative corrections on the external scale $Q$. The magnitude of these power-corrections cannot be calculated, but assuming some universality properties [6, 9] it is possible to estimate it for a large class of observables based on experimental data for one of them.

The SDG approximation, as applied in [7] in the case of the average thrust, provides a systematic framework for the analysis of perturbative running-coupling effects together with the related power-corrections. These two aspects of improving the truncated perturbative result naturally complement each other: they reflect the same physical phenomenon.

It is clear that any observable, including the moments of $1-$ thrust considered here, may eventually depend on other non-perturbative effects, which are unrelated to ambiguities of perturbation theory. We assume that these effects are not important. Since the power-corrections identified from the ambiguities of the perturbative expansion have a definite $Q$ dependence, this assumption can be confronted with experimental data.

It should be emphasized that, contrary to the Operator Product Expansion (OPE), the SDG renormalon approach lacks the rigor of a systematic expansion: there is no small parameter which distinguishes the contribution of multiple emission from that of a single emission. One should be aware of the possibility that in certain cases the leading power-corrections cannot be analyzed in the framework of the SDG calculation as they are associated with soft gluon emission from configurations of three of more hard partons. In particular, in the case of the higher moments of 1 - thrust, such effects may yield $\alpha_{s}\left(Q^{2}\right) / Q$ power-corrections [14, 5, 11, 12]. We shall return to this important issue in the conclusions.

Further subtleties are related to the fact that event-shape variables are not completely inclusive [8, 7]: they are sensitive to certain details of the final state, while the resummation procedure we use is completely inclusive with respect to the fragmentation products of the gluon.

The purpose of this work is to examine, in the framework of the SDG approximation, running-coupling effects and power-corrections to higher moments of $1-$ thrust. We begin, in section 2 by analyzing the thrust distribution. As in [7], where the average thrust was analyzed, we use the "massive" gluon dispersive approach [6]. In this framework resummation as well as parameterization of power-corrections are obtained from a so-called characteristic function. After explaining the main assumptions of the SDG model we calculate the characteristic function for the thrust distribution. We then devote a short discussion to the domain of applicability of the SDG result as a function of the thrust. Next, in section 3 we evaluate the characteristic functions for various moments of 1 - thrust and study their properties. In particular we extract the characteristic invariant mass of gluons contributing to the 
various moments and compare the significance of running-coupling effects to other perturbative contributions. We also quantify the effect of the non-inclusive contribution at the next-to-leading order. Finally, we extract the leading power-corrections implied for the various moments of 1 - thrust by the ambiguity of the perturbative SDG result, and identify the regions of phase-space from which they emerge. The conclusions are given in section 4 .

\section{The thrust distribution in the single dressed gluon model}

The differential cross section with respect to the thrust $d \sigma / d t$ can be calculated in perturbation theory in the single dressed gluon (SDG) approximation as follows

$$
\left.\frac{d \sigma}{d t}(t)\right|_{\mathrm{SDG}}=C_{F} \int_{0}^{1} \frac{d \epsilon}{\epsilon} \bar{a}_{\mathrm{eff}}\left(\epsilon Q^{2}\right) \dot{\mathcal{F}}(\epsilon, t)=C_{F} \int_{0}^{1} \frac{d \epsilon}{\epsilon} \bar{\rho}\left(\epsilon Q^{2}\right)[\mathcal{F}(\epsilon, t)-\mathcal{F}(0, t)]
$$

where the integration over $\epsilon \equiv \mu^{2} / Q^{2}$ corresponds to inclusive summation over final states into which the emitted gluon fragments. In this calculation [6] a final state of invariant mass $\mu^{2}$ is characterized by a single universal (i.e. observable independent) function $\bar{\rho}\left(\mu^{2}\right)$ which is identified as the time-like discontinuity of the coupling $\bar{a}\left(k^{2}\right) \equiv \bar{\alpha}_{s}\left(k^{2}\right) / \pi$, where the bar stands for a specific renormalization-scheme. The two integrals in (2.1) are related by integration by parts:

$$
\dot{\mathcal{F}}(\epsilon, t) \equiv-\epsilon \frac{d}{d \epsilon} \mathcal{F}(\epsilon, t),
$$

and the "time-like coupling" $\bar{a}_{\text {eff }}\left(\mu^{2}\right)$ obeys

$$
\mu^{2} \frac{d \bar{a}_{\text {eff }}\left(\mu^{2}\right)}{d \mu^{2}}=\rho\left(\mu^{2}\right) .
$$

The thrust characteristic function $\mathcal{F}(\epsilon, t)$ is obtained from the following integral over phase-space,

$$
\mathcal{F}(\epsilon, t)=\int_{\text {phase space }} d x_{1} d x_{2} \mathcal{M}\left(x_{1}, x_{2}, \epsilon\right) \delta\left(1-T\left(x_{1}, x_{2}, \epsilon\right)-t\right)
$$

where $C_{F} a \mathcal{M}$ is the squared tree level matrix element for the production of quarkanti-quark pair and a gluon of virtuality $\mu^{2} \equiv \epsilon Q^{2}$, and

$$
\mathcal{M}\left(x_{1}, x_{2}, \epsilon\right)=\frac{1}{2}\left[\frac{\left(x_{1}+\epsilon\right)^{2}+\left(x_{2}+\epsilon\right)^{2}}{\left(1-x_{1}\right)\left(1-x_{2}\right)}-\frac{\epsilon}{\left(1-x_{1}\right)^{2}}-\frac{\epsilon}{\left(1-x_{2}\right)^{2}}\right] .
$$

The integration variables $x_{1,2}$ represent the energy fraction of each of the quarks in the center-of-mass frame. The energy fraction of the gluon is $x_{3}=2-x_{1}-x_{2}$.

For the calculation of the characteristic function we shall use the following [6, 7] definition of the thrust

$$
T=\frac{\sum_{i}\left|\vec{p}_{i} \cdot \vec{n}_{T}\right|}{\sum_{i} E_{i}}=\frac{\sum_{i}\left|\vec{p}_{i} \cdot \vec{n}_{T}\right|}{Q}
$$


In case of three partons (a quark, an anti-quark and a "massive" gluon) it yields [0],

$$
1-T\left(x_{1}, x_{2}, \epsilon\right)=\min \left\{1-x_{1}, 1-x_{2}, 1-\sqrt{\left(2-x_{1}-x_{2}\right)^{2}-4 \epsilon}\right\} .
$$

Note that in the definition (2.6) the denominator is modified [6, 7] with respect to the standard one (1.1), $\sum_{i}\left|\vec{p}_{i}\right| \longrightarrow \sum_{i} E_{i}$, in a way that does not change the observable for massless partons. The virtual ("massive") gluon is understood to fragment eventually into massless partons. This modification ensures that the value of the thrust calculated with a "massive" gluon will be correct, provided that all the (massless) partons produced in the process of the gluon fragmentation end up in the same hemisphere with respect to $\vec{n}_{T}$. The inclusive calculation performed here is justified only if the gluon fragmentation is predominantly collinear, in which case fragmentation into opposite hemispheres will be rare. The discrepancy between the inclusive "massive gluon" calculation and the full non-inclusive calculation was addressed before by several authors [8, 7, 26, 14]. This issue will be further discussed in the next section in the context of the moments of 1 - thrust.

The last ingredient for the calculation of the characteristic function is the phasespace $^{\dagger}$. Fig. 1 shows the three parton phase-space in case of a gluon with a "mass" of $\mu^{2}=\epsilon Q^{2}=0.1 Q^{2}$. The external boundaries of phase-space in the figure correspond to the softest gluons (the upper curved line) and to the hardest ones (the lower linear line). The dashed lines represent the separation of phase-space according to which particle carries the largest momentum and thus determines the thrust axis (cf. eq. (2.7)): in the upper left region $T=x_{2}$, in the upper right region $T=x_{1}$ and in the lower region $T=\sqrt{x_{3}^{2}-4 \epsilon}$.

It is important to note that the two upper regions of phase-space in the figure (where one of the primary quarks carries the largest momentum, $T=x_{1,2}$ ) have three corners with a definite physical meaning for $\epsilon \ll 1$ :

(i) the collinear limit corresponding to a two-jet configuration, with $t=\epsilon$. This is the lowest possible value of $t$, given $\epsilon$.

(ii) the three parton symmetric limit, corresponding to a three-jet configuration, $t=(2 \sqrt{1+3 \epsilon}-1) / 3$. This is the highest possible value of $t$, given $\epsilon$.

(iii) the large-angle soft gluon limit, with $t=\sqrt{\epsilon}$. Here gluons are emitted close to 90 degrees with respect to the quark-anti-quark direction.

It is clear from fig. 1 that in order to perform the integral in (2.4) one has to treat separately values of $t$ smaller than $\sqrt{\epsilon}$ where the soft gluon phase-space boundary (the curved line in fig. 1) is relevant, vs. $t$ larger than $\sqrt{\epsilon}$, where it is not. As explained above, the value $t \simeq \sqrt{\epsilon}$ corresponds, for small $\epsilon$, to the situation where

\footnotetext{
${ }^{\dagger}$ The reader is referred to [7] for more details on the calculation of the phase-space boundaries.
} 


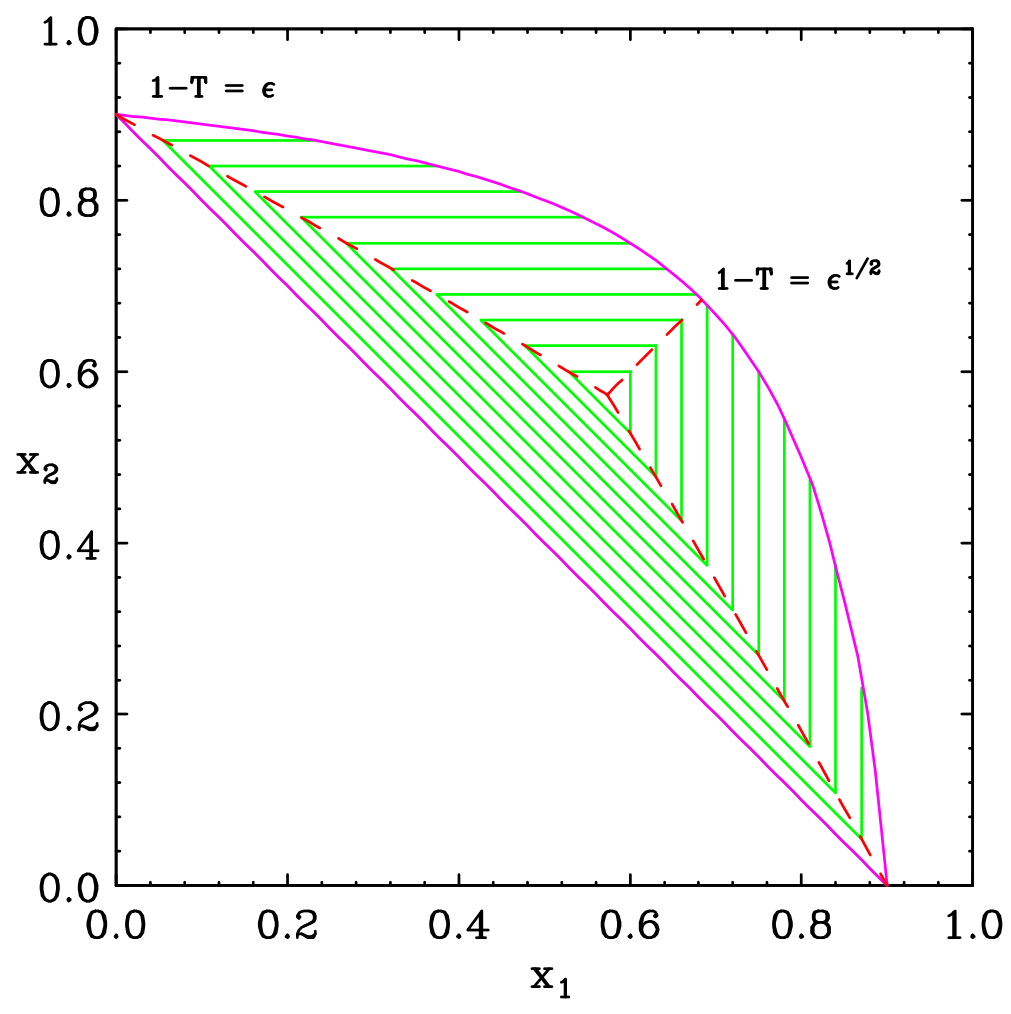

Figure 1: Phase-space for the emission of a "massive" gluon with $\mu^{2}=\epsilon Q^{2}=0.1 Q^{2}$ in the plane of the quark and anti-quark energy fractions $\left(x_{1,2}\right)$. The outer continuous lines represent phase-space limits, the dashed lines separate the phase-space according to the identification of the thrust axis $\vec{n}_{T}$ and internal continuous lines are constant thrust contours. These contours are drawn with separation of $\Delta T=0.03$, stating at the lowest possible value of $1-T=\epsilon=0.1$ where two of the three partons are roughly collinear, up to the highest possible value of $1-T=(2 \sqrt{1+3 \epsilon}-1) / 3 \simeq 0.427$ where the momentum splits equally between the three partons.

the gluon is soft and emitted in a large angle with respect to the quark-anti-quark direction. This part of phase-space is the source of the $1 / Q$ power-corrections for the average thrust, and as we shall see in the next section, also for the leading, though much suppressed, infrared power-corrections for higher moments of 1 - thrust, at the SDG level. Note that other limits of phase-space, as well as the squared matrix element (2.5) itself do not contain any $\sqrt{\epsilon}$ terms. In particular, in the collinear limit $t \simeq \epsilon$.

Evaluating (2.4) we obtain the characteristic function for the thrust distribution,

$$
\mathcal{F}(\epsilon, t)= \begin{cases}\mathcal{F}_{Q}^{l}(\epsilon, t)+\mathcal{F}_{G}(\epsilon, t) & \epsilon<t<\sqrt{\epsilon} \\ \mathcal{F}_{Q}^{h}(\epsilon, t)+\mathcal{F}_{G}(\epsilon, t) & \sqrt{\epsilon}<t<\frac{2}{3} \sqrt{1+3 \epsilon}-\frac{1}{3}\end{cases}
$$

where the dominant contribution $\mathcal{F}_{Q}(\epsilon, t)$ corresponds to the phase-space regions 
where one of the primary quarks carries the largest momentum $\left(T=x_{1,2}\right)$ and $\mathcal{F}_{G}(\epsilon, t)$ corresponds to the region where the gluon momentum is the largest (see fig. (1). The superscripts $l$ and $h$ on $\mathcal{F}_{Q}(\epsilon, t)$ denote low and high $t$ values, respectively. These functions are given by

$$
\begin{aligned}
\mathcal{F}_{Q}^{h}(\epsilon, t) & =-\frac{1}{t}\left[(1-t+\epsilon)^{2}+(1+\epsilon)^{2}\right] \ln \frac{t}{q-t}+\left(3-2 \frac{q}{t}+\frac{1}{2} \frac{1}{t}+\frac{1}{2} t-q\right) \\
& +\left(4-2 \frac{q}{t}+3 \frac{1}{t}-\frac{q}{t^{2}}+\frac{1}{q-t}\right) \epsilon \\
\mathcal{F}_{Q}^{l}(\epsilon, t) & =-\frac{1}{t}\left[(1-t+\epsilon)^{2}+(1+\epsilon)^{2}\right] \ln \frac{\epsilon}{t(q-t)}+\left(1-2 \frac{q}{t}+\frac{1}{2} \frac{1}{t}-q\right) \\
& +\left(3 \frac{1}{t}+\frac{1}{q-t}-\frac{q}{t^{2}}+2 \frac{1}{t^{2}}+2-2 \frac{q}{t}\right) \epsilon+\left(2+\frac{1}{2 t}\right) \frac{\epsilon^{2}}{t^{2}} \\
\mathcal{F}_{G}(\epsilon, t) & =\frac{1-t}{q^{2}}\left[\left((1+\epsilon)^{2}+(1+\epsilon-q)^{2}\right) \ln \frac{q-t}{t}+(2 t-q)\left(q+\frac{\epsilon}{t}+\frac{\epsilon}{q-t}\right)\right]
\end{aligned}
$$

where $q \equiv \sqrt{(1-t)^{2}+4 \epsilon}$.

Eq. (2.1) with $\mathcal{F}(\epsilon, t)$ given by (2.8) and (2.9) can now be used to calculate the SDG contribution to the thrust distribution. The leading order perturbative result [27 can be recovered from (2.1) by assuming a constant coupling $\bar{a}_{\text {eff }}\left(\mu^{2}\right) \simeq$ const. This yields

$$
\left.\frac{d \sigma}{d t}(t)\right|_{\mathrm{LO}}=C_{F} \bar{a}_{\mathrm{eff}}\left(\mu^{2}\right) \int_{0}^{1} \frac{d \epsilon}{\epsilon} \dot{\mathcal{F}}(\epsilon, t)=C_{F} \bar{a}_{\mathrm{eff}}\left(\mu^{2}\right) \mathcal{F}(0, t)
$$

with

$$
\mathcal{F}(0, t)=\frac{3 t^{2}-3 t+2}{t(1-t)} \ln \frac{1-2 t}{t}-\frac{3}{2} \frac{(1+t)(1-3 t)}{t}
$$

for $t \leq 1 / 3$. Eq. (2.10) is the contribution to the thrust distribution of an on-shell gluon. The improved calculation (2.1) takes into account the emission of "massive" gluons that later dissociate. For a given thrust value, the gluon virtuality $\mu^{2}=\epsilon Q^{2}$ can change in the following range:

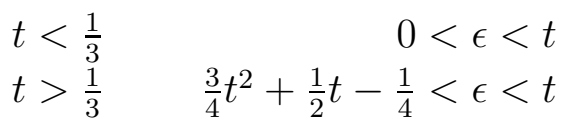

Whereas the leading order result (2.10) depends on an a priori arbitrary choice of the renormalization scale $\mu^{2}$, eq. (2.1) resums running coupling effects to all orders, and is therefore renormalization-group invariant ${ }^{\ddagger}$. Note that performing the integral

\footnotetext{
${ }^{\ddagger}$ In fact, to have full renormalization-group invariance we have to fix the scheme of $\bar{a}$. This requires further assumption concerning the diagrams that contribute to the gluon fragmentation ("dressing the gluon" [7, 24]). Below we fix it according to the Abelian-limit, in order to guarantee the correct resummation of the terms leading in $\beta_{0}$.
} 
in eq. (2.1) can be in turn re-formulated as a specific choice of the renormalization point $\mu$ in (2.10) according to the BLM criterion [25]. For the thrust distribution this representation seems rather cumbersome, since the BLM scale will depend on $t$ in a complicated way. We shall use the BLM formulation later on for the moments of 1 - thrust.

In spite of the resummation achieved by eq. (2.1), since this calculation takes into account just a single gluon emission its applicability is limited as follows:

(i) At "high" values 1 - thrust, $t \gtrsim 1 / 3$, only non-planar configurations, e.g. more than three hadron jets, contribute. The leading-order perturbative result (2.10) corresponding to three partons vanishes identically for $t \geq 1 / 3$ and the first non-vanishing contribution appears at the next order $\mathcal{O}\left(a^{2}\right)$. The resummation result (2.1) does not vanish above $t=1 / 3$. This fits the intuitive expectation: the fragmentation of the "massive" gluon is not restricted to the three parton plane. On the other hand, it is clear that in this region emission of two hard gluons from the primary quarks is important.

(ii) At low values of 1 - thrust, $C_{F} a \ln ^{2}(1 / t) \gtrsim 1$, the contribution of multiple emission of gluons which are both soft and collinear becomes dominant and must be resummed to all-orders in perturbation theory 18. At even lower 1 - thrust values, $\beta_{0} a \ln (1 / t) \simeq 1$, this resummation breaks down since nonperturbative soft gluon emission becomes important.

In view of these facts, we can expect the SDG approximation to describe the thrust distribution only in some restricted range of intermediate thrust values. Since soft and collinear gluon resummation [18] is complementary to dressing the gluon (2.1), the two resummation procedures can be combined to describe the thrust distribution in a wider range. The most promising approach to describe the differential distribution of event-shape variables in the Sudakov region (here small $t$ ) is based on introducing a non-perturbative "shape-function" (SF) [23, 10, 11, 12]. The physical distribution is then obtained by convoluting this function with the perturbative result. Further analysis of the thrust distribution along these lines will appear in a separate publication. Here we proceed by analyzing moments of 1 - thrust.

\section{Moments of $1-$ thrust}

Resummation of running-coupling effects in the SDG approximation was already demonstrated to be important for the average thrust. In particular, it was shown in [7] that the resummation modifies significantly the value of $\alpha_{s}$ extracted from experimental data. The application of the SDG approximation to the average thrust was justified by asserting that low $t=1-T$ values are suppressed in the average, and therefore the effect of multiple soft and collinear gluons should not be significant. 
From this respect the calculation of higher moments of $1-$ thrust, $<t^{m}>$, based on a SDG is even safer since they have a stronger suppression of the low $t$ region. On the other hand, the contribution to $\left\langle t^{m}\right\rangle$ from extremely high values of $1-$ thrust, $t \gtrsim 1 / 3$, becomes more significant as $m$ increases. For high enough $m$ this contribution must be dominant, making the SDG calculation unreliable.

Let us now assume that the contribution to $\left\langle t^{m}\right\rangle$ is dominated by intermediate values of 1 - thrust where the SDG approximation to the distribution (eq. (2.1)) holds, and use it to calculate the $m$-th moment of 1 - thrust according to

$$
\begin{aligned}
<t^{m}>\sim<t^{m}>_{\mathrm{SDG}} & =\left.\int \frac{d \sigma}{d t}(t)\right|_{\mathrm{SDG}} t^{m} d t=C_{F} \int t^{m} d t \int_{0}^{1} \frac{d \epsilon}{\epsilon} \bar{a}_{\mathrm{eff}}\left(\epsilon Q^{2}\right) \dot{\mathcal{F}}(\epsilon, t) \\
& =C_{F} \int_{0}^{1} \frac{d \epsilon}{\epsilon} \bar{a}_{\mathrm{eff}}\left(\epsilon Q^{2}\right) \dot{\mathcal{F}}_{<t^{m}>}(\epsilon),
\end{aligned}
$$

where in the last step we changed the order of integration defining

$$
\mathcal{F}_{<t^{m}>} \equiv \int \mathcal{F}(\epsilon, t) t^{m} d t
$$

In order to use the resummation formula (3.1) we have to specify the time-like coupling $\bar{a}_{\text {eff }}\left(\mu^{2}\right)$. As explained in [7, 24], the renormalization-scheme defining the coupling $\bar{a}$ should be uniquely determined once an Abelian-like skeleton expansion is established in QCD. For the purpose of the current investigation we shall just use the one-loop approximation of the space-like coupling

$$
\bar{a}\left(k^{2}\right)=\frac{1}{\beta_{0}} \frac{1}{\ln \left(k^{2} / \bar{\Lambda}^{2}\right)}
$$

with $\bar{\Lambda}$ set such that $\bar{a}$ coincides with the Gell-Mann Low effective charge in the Abelian (large $\beta_{0}$ ) limit. This is realized, for example, if $\bar{a}$ is related to the $\overline{\mathrm{MS}}$ coupling by a scale-shift, i.e.

$$
\bar{a}\left(k^{2}\right)=\frac{a_{\overline{\mathrm{MS}}}\left(k^{2}\right)}{1-\frac{5}{3} \beta_{0} a_{\overline{\mathrm{MS}}}\left(k^{2}\right)} .
$$

The corresponding time-like coupling is given by [7]

$$
\bar{a}_{\mathrm{eff}}\left(\mu^{2}\right)=\frac{1}{\beta_{0}}\left[\frac{1}{2}-\frac{1}{\pi} \arctan \left(\frac{1}{\pi} \log \frac{\mu^{2}}{\bar{\Lambda}^{2}}\right)\right] .
$$

Using this coupling in (3.1), the terms which are leading in $\beta_{0}$ will be resummed correctly (ignoring the non-inclusive nature of the observable [8, 7, 26], which will be discussed below), while other terms which are sub-leading in $\beta_{0}$ will be neglected.

Before proceeding with the SDG analysis it is worthwhile to examine the known next-to-leading order result [19]. The next-to-leading order coefficient is calculated 
based on numerical integration over the three and four parton phase-space [20, 21].

In $\overline{\mathrm{MS}}$ it is given by [17, 28]

$$
\begin{aligned}
& <t>=0.7888 C_{F} a_{\overline{\mathrm{MS}}}\left(Q^{2}\right)+\left(-1.1570 C_{F}^{2}+4.4708 C_{F} C_{A}-0.8445 C_{F} N_{f}\right) a_{\overline{\mathrm{MS}}}^{2}\left(Q^{2}\right) \\
& <t^{2}>=0.0713 C_{F} a_{\overline{\mathrm{MS}}}\left(Q^{2}\right)+\left(0.3073 C_{F}^{2}+0.3280 C_{F} C_{A}-0.0583 C_{F} N_{f}\right) a_{\overline{\mathrm{MS}}}^{2}\left(Q^{2}\right) \\
& <t^{3}>=0.0112 C_{F} a_{\overline{\mathrm{MS}}}\left(Q^{2}\right)+\left(0.06877 C_{F}^{2}+0.04973 C_{F} C_{A}-0.00808 C_{F} N_{f}\right) a_{\overline{\mathrm{MS}}}\left(Q^{2}\right) \\
& <t^{4}>=0.0022 C_{F} a_{\overline{\mathrm{MS}}}\left(Q^{2}\right)+\left(0.01622 C_{F}^{2}+0.00989 C_{F} C_{A}-0.00145 C_{F} N_{f}\right) a_{\overline{\mathrm{MS}}}^{2}\left(Q^{2}\right)
\end{aligned}
$$

In order to correctly identify the terms that originate from the running-coupling [7, 24], we use (3.4) to translate (3.6) to the $\bar{a}$ scheme. Then, expressing the $N_{f}$ dependence of the next-to-leading order coefficients in terms of $\beta_{0}=(11 / 12) C_{A}-(1 / 6) N_{f}$ as done in the "naive non-Abelianization" procedure [29] we obtain

$$
\begin{aligned}
<t> & =C_{F}\left[0.7888 \bar{a}\left(Q^{2}\right)+\left(3.7526 \beta_{0}-1.1567 C_{F}-0.1740 C_{A}\right) \bar{a}^{2}\left(Q^{2}\right)\right] \\
<t^{2}> & =C_{F}\left[0.0713 \bar{a}\left(Q^{2}\right)+\left(0.2308 \beta_{0}+0.3073 C_{F}+0.00762 C_{A}\right) \bar{a}^{2}\left(Q^{2}\right)\right] \\
<t^{3}> & =C_{F}\left[0.0112 \bar{a}\left(Q^{2}\right)+\left(0.02981 \beta_{0}+0.06877 C_{F}+0.005271 C_{A}\right) \bar{a}^{2}\left(Q^{2}\right)\right] \\
<t^{4}> & =C_{F}\left[0.0022 \bar{a}\left(Q^{2}\right)+\left(0.005045 \beta_{0}+0.01622 C_{F}+0.00191 C_{A}\right) \bar{a}^{2}\left(Q^{2}\right)\right]
\end{aligned}
$$

Let us now look at the relative magnitudes of the different terms in the next-toleading order coefficients in (3.7). Substituting the QCD values of $C_{F}, C_{A}$ and $\beta_{0}$ (for $N_{f}=5$ ) we obtain the numerical values summarized in table 1. We find that the

\begin{tabular}{|c||c|c|c|c|}
\hline & $<t>$ & $<t^{2}>$ & $<t^{3}>$ & $<t^{4}>$ \\
\hline \hline$\beta_{0}$ & 7.1925 & 0.4423 & 0.0571 & 0.00967 \\
$C_{F}$ & -1.542 & 0.4098 & 0.0917 & 0.02163 \\
$C_{A}$ & -0.522 & 0.0229 & 0.0158 & 0.00574 \\
\hline
\end{tabular}

Table 1: Decomposition of the next-to-leading order coefficient multiplying $C_{F} \bar{a}^{2}\left(Q^{2}\right)$ in eq. (3.7) for each of the moments of $t \equiv 1-T$. Here we substituted $C_{A}=3, C_{F}=4 / 3$ and $N_{f}=5$.

non-Abelian $C_{F} C_{A}$ component is always small. The relative significance of the $C_{F} \beta_{0}$ term associated with the running-coupling decreases with $m$ : this term is absolutely dominant in the case of the average thrust. It is still the largest in the case of $\left\langle t^{2}\right\rangle$, but it is no longer dominant. This trend continues for higher moments $\left\langle t^{m}\right\rangle, m=3$ and 4 . On the other hand, the significance of the double emission term, proportional to $C_{F}{ }^{2}$, increases with $m$. This fits the intuitive expectation: higher moments of $1-$ thrust become sensitive to spherical configurations, and in particular, to multi-jet 
configurations. This property, which makes the next-to-leading order perturbative series less reliable as $m$ increases, also implies that the significance of the resummation of running-coupling effects by the SDG formula (3.1) compared to other contributions decreases with $m$. Note, however, that with increasing orders in perturbation theory, the terms associated with the running-coupling are expected in general to increase fast and eventually dominate the coefficients.

We now want to calculate the characteristic functions (3.2) for the first few moments. Using eq. (2.8) we have

$$
\mathcal{F}_{<t^{m}>}(\epsilon)=\int_{\epsilon}^{\frac{2}{3} \sqrt{1+3 \epsilon}-\frac{1}{3}} \mathcal{F}_{G}(\epsilon, t) t^{m} d t+\int_{\epsilon}^{\sqrt{\epsilon}} \mathcal{F}_{Q}^{l}(\epsilon, t) t^{m} d t+\int_{\sqrt{\epsilon}}^{\frac{2}{3} \sqrt{1+3 \epsilon}-\frac{1}{3}} \mathcal{F}_{Q}^{h}(\epsilon, t) t^{m} d t
$$

or, after rearranging the terms,

$$
\mathcal{F}_{<t^{m}>}(\epsilon)=\int_{\epsilon}^{\frac{2}{3} \sqrt{1+3 \epsilon}-\frac{1}{3}}\left[\mathcal{F}_{G}(\epsilon, t)+\mathcal{F}_{Q}^{h}(\epsilon, t)\right] t^{m} d t-\int_{\epsilon}^{\sqrt{\epsilon}}\left[\mathcal{F}_{Q}^{h}(\epsilon, t)-\mathcal{F}_{Q}^{l}(\epsilon, t)\right] t^{m} d t
$$

where the difference

$\mathcal{F}_{Q}^{h}(\epsilon, t)-\mathcal{F}_{Q}^{l}(\epsilon, t)=-\frac{1}{t}\left[(1-t+\epsilon)^{2}+(1+\epsilon)^{2}\right] \ln \frac{t^{2}}{\epsilon}+2+\frac{t}{2}+\epsilon\left(2-\frac{2}{t^{2}}\right)-\frac{\epsilon^{2}}{t^{2}}\left(2+\frac{1}{2 t}\right)$

contains the essential information on the region $t \simeq \sqrt{\epsilon}$. As we shall see below, non-analytic terms in the expansion of $\mathcal{F}_{<t^{m}>}(\epsilon)$ at small $\epsilon$ imply power-suppressed contributions to $\left\langle t^{m}\right\rangle$. It is the $\sqrt{\epsilon}$ in the upper boundary in the second term of (3.9), corresponding to large-angle soft-gluon emission, which is the source the leading non-analytic terms.

The resulting characteristic functions for $m=1$ and 2 are shown in fig. 2 and these for $m=3$ and 4 - in fig. 3. On this basis, the SDG perturbative sum in (3.1) can be calculated. As an alternative to evaluating the integral we can expand the coupling $\bar{a}_{\text {eff }}\left(\epsilon Q^{2}\right)$ under the integration sign, e.g. in terms for $\bar{a}\left(Q^{2}\right)$, obtaining,

$$
<t^{m}>_{\mathrm{SDG}}=C_{F}\left[d_{0} \bar{a}\left(Q^{2}\right)+d_{1} \beta_{0} \bar{a}^{2}\left(Q^{2}\right)+\left(d_{2}-\frac{\pi^{2}}{3} d_{0}\right) \beta_{0}^{2} \bar{a}^{3}\left(Q^{2}\right)+\cdots\right],
$$

where the coefficients are expressed in terms of the log-moments of the corresponding characteristic function, $d_{0} \equiv \mathcal{F}_{<t^{m}>}(0)$ and

$$
d_{i} \equiv \int_{0}^{1} \dot{\mathcal{F}}_{<t^{m}>}(\epsilon)(-\ln \epsilon)^{i} \frac{d \epsilon}{\epsilon} .
$$

The log-moments can be evaluated by a straightforward numerical integration. The resulting values are summarized in table 2. In the cases of the average thrust and $\left\langle t^{2}\right\rangle$ one identifies already at the first few orders the characteristics of infrared renormalons, namely fast increase (that asymptotically becomes factorial) and a constant sign pattern. This behavior is much delayed for the higher moments. 

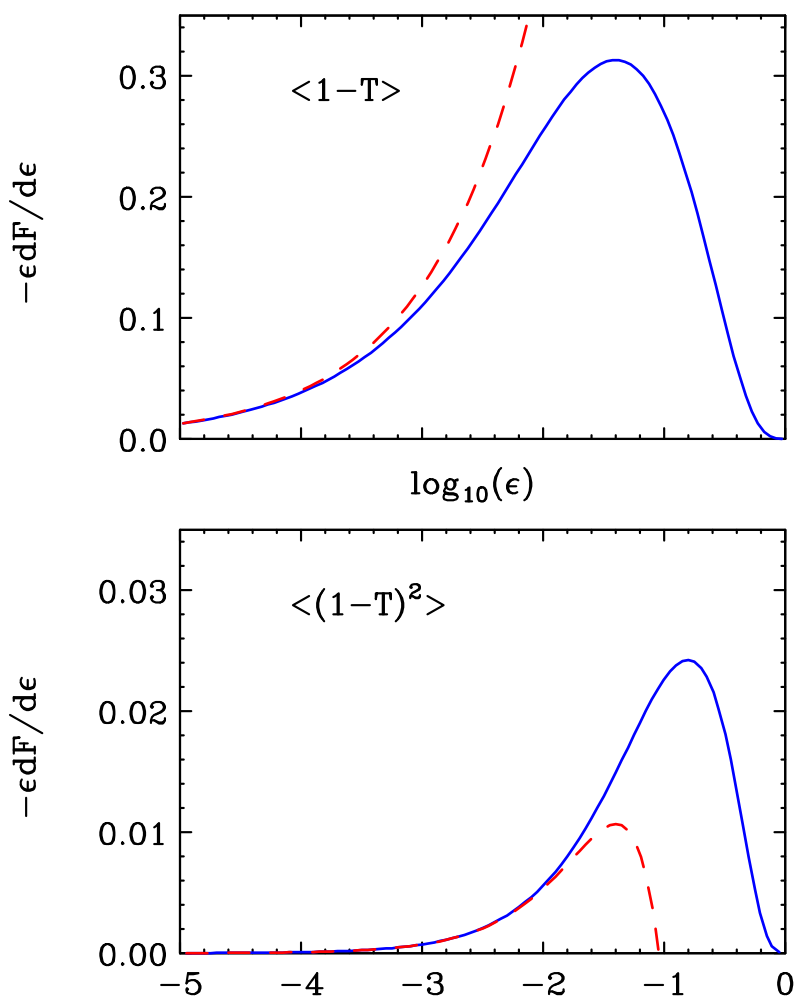

Figure 2: The derivative of the characteristic functions $\dot{\mathcal{F}}(\epsilon)$ for $<1-T>$ (upper plot) and $<(1-T)^{2}>$ (lower plot) as a function of $\log _{10}(\epsilon)$, where $\mu^{2}=\epsilon Q^{2}$ is the gluon virtuality. The dashed lines in the two plots represent the $\mathcal{O}\left(\epsilon^{1 / 2}\right)$ and $\mathcal{O}\left(\epsilon^{3 / 2}\right)$ approximations to $\dot{\mathcal{F}}(\epsilon)$, respectively.

\begin{tabular}{|c||c|c|c|c|}
\hline & $<t>$ & $<t^{2}>$ & $<t^{3}>$ & $<t^{4}>$ \\
\hline \hline$d_{0}$ & 0.7888 & 0.0713 & 0.0112 & 0.0022 \\
$d_{1}$ & 3.588 & 0.1875 & 0.0160 & 0.000139 \\
$d_{2}$ & 21.06 & 0.6296 & 0.0218 & -0.00903 \\
$d_{3}$ & 154.03 & 2.5922 & -0.0020 & -0.0555 \\
$d_{4}$ & 1368.38 & 12.721 & -0.3254 & -0.3108 \\
$d_{5}$ & 14464.59 & 72.835 & -3.1238 & -1.8546 \\
\hline
\end{tabular}

Table 2: First few log-moment of the characteristic functions for various moments of $t \equiv 1-T$.

The approximation of $\left\langle t^{m}>\right.$ by $\left\langle t^{m}\right\rangle_{\mathrm{SDG}}$ in (3.1), or by (3.10), can be improved in a straightforward manner by matching it with the next-to-leading order result (3.7). Such a procedure was used in [7] for the phenomenological analysis of the average thrust. The additional terms in (3.7), correspond primarily to double 

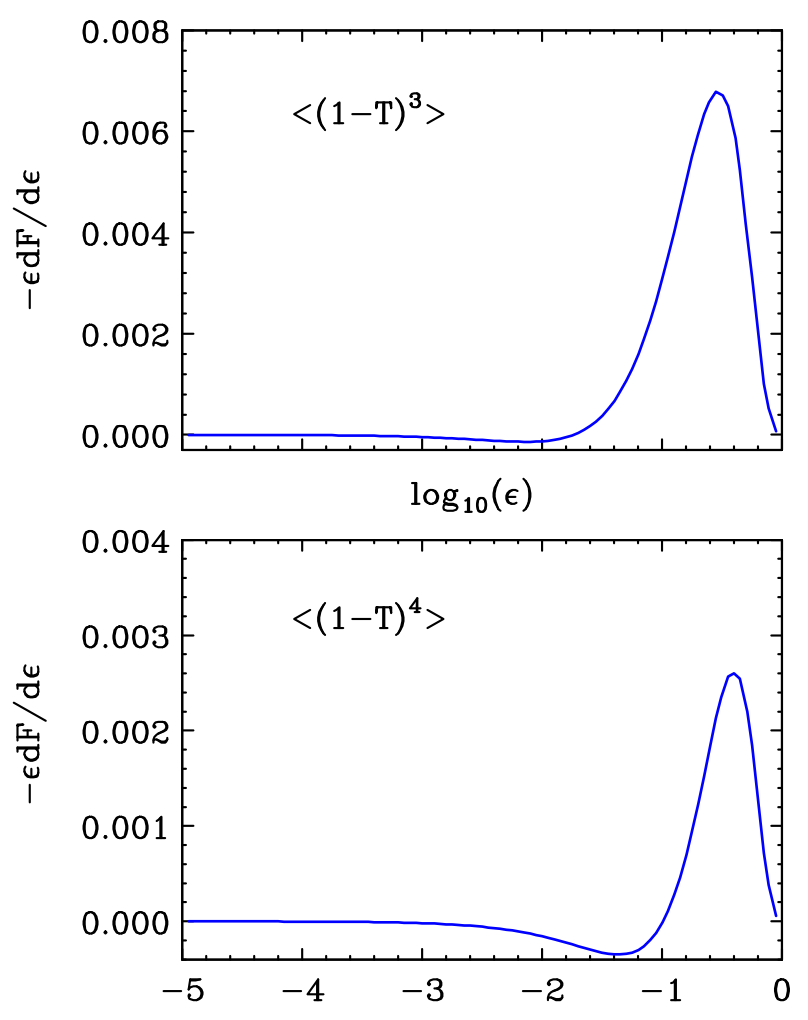

Figure 3: The derivative of the characteristic functions $\dot{\mathcal{F}}(\epsilon)$ for $<(1-T)^{3}>$ (upper plot) and $<(1-T)^{4}>$ (lower plot) as a function of $\log _{10}(\epsilon)$, where $\mu^{2}=\epsilon Q^{2}$ is the gluon virtuality.

gluon emission (a $C_{F}^{2}$ term). They also include a non-Abelian $C_{A} C_{F}$ component, which depends [7, 24] on the identification of the coupling $\bar{a}$ beyond the Abelian limit, and finally, also some residual $\beta_{0}$ dependent piece, which is related [7] to the non-inclusive nature of the thrust.

Had the moments of the thrust been completely inclusive with respect to the fragmentation products of the gluon, the terms proportional to $\beta_{0}$ should have been fully contained in the SDG result (3.1) or (3.10). As explained in section 5.2 in [0], in the inclusive case the $\log$-moments $d_{i}$, for any $i$, are equal to the terms leading in $\beta_{0}$ in the exact calculation. In the present case the next-to-leading order coefficient $d_{1}$ calculated as a log-moment of the inclusive characteristic function differs from the actual $\beta_{0}$ dependent term in (3.7). The "massive gluon" inclusive treatment is justified only if the discrepancy between them is small. In the case of $\langle t\rangle$ this discrepancy was found [0] to be tiny. Comparing table 2 a with eq. (3.7) we find that it is only $4.4 \%$ ! Evidently it increases for the higher moments of 1 - thrust. For $<t^{2}>$ the inclusive approximation still seems reasonable: the discrepancy is $18.7 \%$. It is much worse for $\left\langle t^{3}\right\rangle$, where the discrepancy is $46 \%$ and it completely breaks down for $<t^{4}>$. 
The physical reason why the inclusive calculation for the high moments fails is that $\left\langle t^{m}>\right.$ becomes sensitive to large $t$ values. The high $t$ region is correlated with high gluon virtuality (see e.g. (2.12)). High virtuality allows the gluon fragmentation products to spread in large angles compared to the original gluon momentum. Consequently, fragmentation into opposite hemispheres becomes more probable. In such a case, a full non-inclusive calculation would yield a higher thrust $(T)$ value compared to the inclusive calculation that takes into account the gluon momentum itself (this follows from (2.6) using the triangle inequality). As a result, the inclusive calculation under-estimates the thrust distribution $d \sigma / d t$ at large $t$ on the expense of over-estimating it for somewhat lower $t$ values. This is why for all the moments the full $\beta_{0}$ dependent term in (3.7) is larger than the inclusive result $d_{1}$. The success of the inclusive approximation for the average thrust and for $\left\langle t^{2}\right\rangle$ is related to the fact that most of the gluon fragmentation is roughly collinear.

For the first two moments of 1 - thrust, the coefficients in (3.10) increase fast due to infrared renormalons already at the first few orders. It is clear that $\bar{a}\left(Q^{2}\right)$ is not a good expansion parameter. A simple way to approximate the perturbative sum (ignoring the renormalon ambiguity) is the BLM method. In this case we approximate (3.1) by

$$
<t^{m}>_{\mathrm{SDG}} \simeq C_{F} \bar{a}_{\mathrm{eff}}\left(\mu_{\mathrm{BLM}}^{2}\right) \int_{0}^{1} \frac{d \epsilon}{\epsilon} \dot{\mathcal{F}}_{<t^{m}>}(\epsilon)=C_{F} \bar{a}_{\mathrm{eff}}\left(\mu_{\mathrm{BLM}}^{2}\right) \mathcal{F}_{<t^{m}>}(0)
$$

where, at leading order, the BLM scale is the center of the characteristic function, i.e. the average virtuality of the gluon contributing to $\left\langle t^{m}\right\rangle_{\mathrm{SDG}}$,

$$
\mu_{\mathrm{BLM}}^{2}=Q^{2} \exp \left(-\int_{0}^{1} \dot{\mathcal{F}}_{<t^{m}>}(\epsilon)(-\ln \epsilon) \frac{d \epsilon}{\epsilon} / \int_{0}^{1} \dot{\mathcal{F}}_{<t^{m}>}(\epsilon) \frac{d \epsilon}{\epsilon}\right) \equiv Q^{2} \exp \left(-d_{1} / d_{0}\right) .
$$

In principle, higher order log-moments (3.11) can be used to improve the approximation in (3.12) by further modifying the scale (see, for example, eq. (33) in [24]). For the qualitative discussion here, the leading-order BLM approximation will suffice. Using (3.4) one can translate the BLM result to the $\overline{\mathrm{MS}}$ scheme,

$$
<t^{m}>_{\mathrm{SDG}} \simeq C_{F} a_{\overline{\mathrm{MS}}}\left(\mu_{\mathrm{BLM}, \overline{\mathrm{MS}}}^{2}\right) \mathcal{F}_{<t^{m}>}(0)
$$

with $\mu_{\mathrm{BLM}, \overline{\mathrm{MS}}}^{2}=\mu_{\mathrm{BLM}}^{2} e^{-5 / 3}$.

The first two log-moments in table 2 allow to calculate the leading-order BLM scales for the various moments of $1-$ thrust according to (3.13). The results are shown in table 3. The numbers in the first row of the table have the interpretation of the average gluon virtuality contributing to $<t^{m}>$. We see that

(i) the typical virtuality, which is also the correct argument for the coupling in (3.12), is much lower than $Q$ for the first moments of 1 - thrust, especially for the average thrust and for $<t^{2}>$. 


\begin{tabular}{|c||c|c|c|c|}
\hline & $<t>$ & $<t^{2}>$ & $<t^{3}>$ & $<t^{4}>$ \\
\hline \hline$\mu_{\text {BLM }} / Q$ & 0.1028 & 0.2685 & 0.4889 & 0.9689 \\
$\mu_{\text {BLM }, \overline{\mathrm{MS}}} / Q$ & 0.0447 & 0.1167 & 0.2125 & 0.4211 \\
\hline
\end{tabular}

Table 3: BLM scales in the "skeleton scheme" and in $\overline{\mathrm{MS}}$ for various moments of $t \equiv 1-T$.

(ii) with increasing $m$ highly virtual gluons become dominant (at large $m, \mu_{\mathrm{BLM}} \longrightarrow Q$ ).

These features can be read directly from the characteristic function curves. In fig. 2 we see that $\mathcal{F}_{\langle t\rangle}(\epsilon)$ is wide and centered at very low virtualities while $\mathcal{F}_{\left\langle t^{2}\right\rangle}(\epsilon)$ is narrower and centered at higher virtualities. Fig. 8 shows that this trend persists for the higher moments. Note that $\mathcal{F}_{<t^{m}>}(\epsilon)$ for $m \geq 3$, as opposed to $m=1$ and 2 , is not positive definite. Thus for $m \geq 3$ the interpretation of $\mu_{\text {BLM }}$ as the average gluon virtuality contributing to $\left\langle t^{m}\right\rangle$ is no longer accurate. Note that for $m=4$ the cancelation between positive and negative contributions to $d_{1}$ is already large, making the approximation of the integral (3.1) by the coupling at the BLM scale absolutely unreliable.

Let us now turn to discuss the power-correction implied by the SDG perturbative sum. The starting point is the observation that eq. (3.1), understood as an all-order perturbative sum, is ill-defined due to infrared renormalons. The standard way to resum asymptotic series of this type is the Borel method. To obtain a Borel representation one expresses the coupling in (3.1) as

$$
\bar{a}_{\mathrm{eff}}^{\mathrm{PT}}\left(\mu^{2}\right)=\frac{1}{\beta_{0}} \int_{0}^{\infty} d z \bar{a}(z) \frac{\sin \pi z}{\pi z} e^{-z \ln \frac{\mu^{2}}{\Lambda^{2}}},
$$

where the sin factor arises from the analytic continuation to the time-like region. For the one-loop coupling (3.3), $\bar{a}(z)=1$. Substituting this in (3.1) and changing the order of integration one arrives at the following Borel-sum,

$$
<t^{m}>_{\mathrm{SDG}}=\frac{C_{F}}{\beta_{0}} \int_{0}^{\infty} d z B_{<t^{m}>}(z) e^{-z \ln \frac{Q^{2}}{\Lambda^{2}}}
$$

with

$$
B_{<t^{m}>}(z)=\frac{\sin \pi z}{\pi z} \int_{0}^{1} \epsilon^{-z} \dot{\mathcal{F}}_{<t^{m}>}(\epsilon) \frac{d \epsilon}{\epsilon} .
$$

The integral over $z$ in (3.16) is ill-defined yielding an ambiguous result (see [7, 30] for more details). The physical reason for this ambiguity is that our "perturbative" calculation (3.1) actually depends on the coupling at all scales, including the infrared. This ambiguity can thus be resolved only at the non-perturbative level. Nevertheless, in practice it is possible [7] to cure the ambiguity by defining the perturbative sum through some regularization procedure, like the principal-value or a momentum cutoff. At the next step, one adds to the regularized perturbative sum 
explicit power-suppressed terms that have the same dependence on $Q^{2}$ as the leading ambiguities and a normalization which is controlled by free parameters to be fixed by a fit. An attractive possibility [6] is to write this parameterization in terms of the small $k^{2}$ moments of coupling $\bar{a}\left(k^{2}\right)$, assumed to be regular in the infrared at the non-perturbative level. Thanks to the assumed universality of $\bar{a}\left(k^{2}\right)$, this parameterization immediately implies universality of the power suppressed terms for different observables, up to calculable factors which depend on the characteristic function.

Notice that since the time-like coupling (3.5) is finite for any $\mu^{2}$ and has an infrared fixed-point: $\bar{a}_{\text {eff }}(0)=1 / \beta_{0}$, the integral (3.1) with (3.5) is well-defined. This is the so-called 31 "Analytic Perturbation Theory" (APT) result. It differs by ambiguous power terms ${ }^{\S}$ from the corresponding Borel-sum (3.16). The APT result, just like the principal-value Borel-sum, is a specific regularization of the perturbative sum. Its advantage is that it is straightforward to calculate.

In general, if we ignore sub-leading perturbative terms that are not included in the SDG approximation (3.1), the physical result should be given by the regularized perturbative sum plus power-corrections. The apparent ambiguity in the choice of the regularization procedure is eliminated by the power terms. In absence of relevant non-perturbative calculation, the only way to appreciate the significance of the power terms is by comparing different regularization procedures.

One has to distinguish between two types of power-suppressed ambiguities of the perturbative sum:

(i) differences between various regularizations, e.g. between the APT integral and the principal-value Borel-sum.

(ii) the specific differences which are associated with infrared scales, i.e. space-like momentum scales at which the coupling is not under control in perturbation theory.

This second type of ambiguity can be studied by introducing a momentum cutoff in the space-like region [30, 7]. It was shown to be related to non-analytic terms [32, 29, 6, 30, 7] in the small gluon virtuality (or $\epsilon$ ) expansion of the characteristic function. In case of observables that admit an operator product expansion (OPE), the parametric dependence of these ambiguous terms on $Q$ should fit the dimensions of the higher-twist operators. On the other hand, the first type of ambiguity may appear due to both analytic and non-analytic terms in the small $\epsilon$ expansion of the characteristic function [30]. This implies that certain regularizations differ from others, as well as from the physical non-perturbative result, by non-infrared non-OPE power terms. The simplest possibility is that non-perturbative effects are

\footnotetext{
${ }^{\S}$ See [7, 30] and refs. therein.

IThis does not apply to moments of 1 - thrust which cannot be expressed in terms of local operators.
} 
restricted to large distances. Then a momentum cutoff in the space-like region as introduced in [30, 7], or equivalently, the principal-value Borel-sum, define a class of favorable regularizations which differ from each other, as well as from the physical result, just by infrared power-corrections. We stress that the APT integral does not belong to this class of regularizations, and is suggestive of a different scenario for the regularization of perturbation theory, advocated in [31].

In order to study the ambiguities of perturbation theory that emerge from the SDG calculation in the case of moments of 1 - thrust (3.1), we calculated the small $\epsilon$ asymptotic expansions of the characteristic functions $\mathcal{F}_{<t^{m}>}(\epsilon)$ given by eq. (3.9). These expansions for $m=1$ through 4 are summarized in the appendix in eq. (A.1) through (A.4) and in table 6. According to the explanation above, non-analytic terms in the expansion of the characteristic functions are of special interest, since they imply ambiguity of perturbation theory which is associated with large distances. There are two physically distinct sources of non-analytic terms contributing to the expansions (A.1) through (A.4). The first is the contribution of collinear soft gluon emission. It originates in the lower integration limit $(t=\epsilon)$ of the two terms in (3.9). The second is large-angle soft-gluon emission, which originates in the upper integration limit $(t=\sqrt{\epsilon})$ of the second term in (3.9). To distinguish between these two regions of phase-space, we evaluated separately the second integral in (3.9) at its upper limit. The results, which represent the contribution of large-angle soft-gluon emission to $\mathcal{F}_{<t^{m}>}(\epsilon)$, are summarized in eq. (A.5). The expansions in the appendix can be used, based on the general formulae obtained in ref. [7], to analyze the power-corrections implied by (3.1).

Note first that all the leading non-analytic terms in the small $\epsilon$ expansion of the characteristic functions are of the half-integer type. These terms, as well as all the other half-integer terms, originate exclusively in the large-angle soft-gluon region through the upper limit $t=\sqrt{\epsilon}$ in the second integral in (3.9). This conclusion follows from the comparison of eq. (A.5) with the full expansion in eq. (A.1) through (A.4).

Note also that in eq. (A.1) through (A.4) there are no logarithmic terms at order $\epsilon \ln \epsilon$. In general, presence of such terms in the characteristic function implies [6, 7] infrared power-corrections that scale as $1 / Q^{2}$. Their absence at the level of the moments of $1-$ thrust, $\left\langle t^{m}\right\rangle$, is not expected a priori, since they are present at the level of the thrust distribution, see eq. (2.8) with (2.9). Comparing eq. (A.5) to eq. (A.1) it becomes apparent that for the average thrust the $\epsilon \ln \epsilon$ terms cancel between collinear and large-angle soft terms. For higher moments of $1-$ thrust such terms do not appear even in the separate contributions of these two phase-space regions (see eq. (A.5)). The first non-vanishing logarithmic terms for the first two moments, $m=1$ and 2, appear at order $\epsilon^{2} \ln \epsilon$, namely $1 / Q^{4}$. These terms originate in both collinear and large-angle soft-gluon regions. The leading logarithmic terms 
for higher moments, $m=3$ and 4, appear much later, and exclusively" due to the collinear limit. Since in non of the moments, do logarithmic terms appear as the leading non-analytic terms we can safely ignore them in the more detailed analysis that follows.

Let us now summarize some formulae [7] for the regularization dependence of (3.1), using the simplest one-loop ansatz (3.3) for the coupling. Given a generic term of the form ${ }^{* *} c_{n} \epsilon^{n}$ in the small $\epsilon$ expansion of $\mathcal{F}_{<t^{m}>}(\epsilon)-\mathcal{F}_{<t^{m}>}(0)$, the difference between the APT regularization and the Borel-sum is given by [29, 30, 7],

$$
\delta<t^{m}>\equiv-c_{n} \frac{C_{F}}{\beta_{0}}\left(\frac{\bar{\Lambda}^{2}}{Q^{2}}\right)^{n} \exp ( \pm i \pi n) .
$$

Note that for half-integer values of $n$, this formula yields an ambiguous imaginary result. This is the ambiguity of the Borel-sum. The principal-value regularization amounts to taking the real part of the Borel sum, and thus it coincides with the APT integral, as far as these terms are concerned. On the other hand for integer values of $n$ the difference between the APT integral and the Borel-sum is unambiguous and real.

To quantify the ambiguities associated with infrared scales we define a space-like momentum cutoff $\mu_{I}$, with $t_{I} \equiv \ln \left(\mu_{I}^{2} / \bar{\Lambda}^{2}\right)$. The contribution to the principal-value Borel-sum from momentum scales below $\mu_{I}$ is given by [7],

$$
<t^{m}>_{\mathrm{IR}} \equiv-c_{n} \frac{C_{F}}{\beta_{0}}\left(\frac{\mu_{I}^{2}}{Q^{2}}\right)^{n} \frac{\sin \pi n}{\pi} \exp \left(-n t_{I}\right) \operatorname{Ei}\left(n t_{I}\right)
$$

In accordance with the general statement above, $\left\langle t^{m}\right\rangle_{\mathrm{IR}}$ is non-zero for non-analytic terms with half-integer $n$ while it vanishes for analytic terms where $n$ is integer.

Using (3.18) and (3.19) with the expansions of the characteristic functions in eq. (A.1) through (A.4) we find the leading ambiguities associated with the perturbative sum (3.1) of each moment of 1 - thrust. The two rows in table 1 correspond to (3.18) and (3.19), respectively. The table shows the parametric dependence of the leading ambiguity on the center-of-mass energy $Q$. Table 5 shows the corresponding numerical values of the power terms for $Q=\mathrm{M}_{\mathrm{Z}}$, normalized by the (approximate) perturbative result (3.12) with the BLM scales of table 3, for $\mu_{I}=2 \mathrm{GeV}$, assuming $\alpha_{s}\left(\mathrm{M}_{\mathrm{Z}}\right)=0.115$ and $N_{f}=5$.

As mentioned above, in all the cases the leading contributions from the infrared (3.19) appear due to half-integer terms associated with large-angle soft-gluon emission. These ambiguities imply existence of non-perturbative power terms which scale as $1 / Q$ for $<t>$, as $1 / Q^{3}$ for $<t^{2}>$ and $<t^{3}>$, and as $1 / Q^{5}$ for $<t^{4}>$.

\footnotetext{
"In eq. A.5 for $m=3$ and 4 there are no logarithmic terms at all.

**As stressed above we ignore logarithmic terms. Regularization of integrals like (3.1) in presence of such terms was also analyzed in [7].
} 


\begin{tabular}{|c||c|c|c|c|}
\hline & $<t>$ & $<t^{2}>$ & $<t^{3}>$ & $<t^{4}>$ \\
\hline \hline$\delta<t^{m}>$ & $1 / Q$ & $1 / Q^{2}$ & $1 / Q^{2}$ & $1 / Q^{2}$ \\
$<t^{m}>_{\text {IR }}$ & $1 / Q$ & $1 / Q^{3}$ & $1 / Q^{3}$ & $1 / Q^{5}$ \\
\hline
\end{tabular}

Table 4: Leading ambiguities in the perturbative calculation for various moments of $t \equiv 1-T$

\begin{tabular}{|c||c|c|c|c|}
\hline & $<t>$ & $<t^{2}>$ & $<t^{3}>$ & $<t^{4}>$ \\
\hline \hline$\delta<t^{m}>/<t^{m}>$ & $\pm 0.075 i$ & $-0.3810^{-3}$ & $0.2210^{-3}$ & $0.3710^{-3}$ \\
$<t^{m}>_{\mathrm{IR}} /<t^{m}>$ & 0.17 & $0.1410^{-3}$ & $-0.2410^{-3}$ & $-0.3510^{-6}$ \\
\hline
\end{tabular}

Table 5: Relative magnitudes of the ambiguities in the perturbative calculation for various moments of $t \equiv 1-T$ at $Q=\mathrm{M}_{\mathrm{Z}}$.

Considering the leading ambiguity of the perturbative sum (3.18), a definite difference exists between the case of the average thrust, $m=1$, and higher moments, $m \geq 2$. In the case of $\langle t\rangle$ the leading ambiguity of the Borel-sum is due to the same $\sqrt{\epsilon}$ term which dominates the infrared contribution, whereas for $\left\langle t^{m}\right\rangle$ with $m \geq 2$ the leading ambiguity originates in an analytic term $\left(c_{1} \epsilon\right)$ in $\mathcal{F}_{<t^{m}>}(\epsilon)$. It is therefore not associated with large distance scales. Moreover, a careful examination of the source of the terms proportional to $\epsilon$, (compare e.g. (A.5) with the full expansion (A.1) through (A.4)) shows that they are not associated with any definite part of phase-space.

The numerical values in table 5 clearly indicate that all the power-corrections that appear in the SDG approximation are small for any $m \geq 2$. The relative error at $\mathrm{M}_{\mathrm{Z}}$ is less than a pro-mil. The case of the average thrust is unique having a significant contribution from the infrared: with the parameters quoted above it is $17 \%$ (!) at $\mathrm{M}_{\mathrm{Z}}$. We stress that in contrast with the average thrust case, the difference between the APT and Borel-sum regularizations for $m \geq 2$ becomes parametrically larger, as well as numerically larger at $\mathrm{M}_{\mathrm{Z}}$, than the infrared contribution to the Borel-sum. This might give an opportunity to use experimental data in order to constrain $1 / Q^{2}$ power terms such as the ones making the APT integral different from the principal-value Borel-sum.

\section{Conclusions}

We started in section 2 by calculating the characteristic function of the thrust distribution, $\mathcal{F}(\epsilon, t)$. The result, summarized by eq. (2.8) and (2.9), forms the basis for resummation of running-coupling effects in the SDG approximation in this case. On 
its own the SDG approximation is expected to describe the physical distribution only in a limited range of thrust values. However, when combined with other techniques [18, 12], the range of applicability of the calculated distribution can be extended, leading to more meaningful comparison with experimental data than available today.

In section 3 we concentrated on the first few moments of $1-$ thrust, $\left\langle t^{m}\right\rangle$, $m=1$ through 4 . The corresponding characteristic functions $\mathcal{F}_{<t^{m}>}(\epsilon)$ were obtained by a straightforward integration of $\mathcal{F}(\epsilon, t)$ and then used to study the properties of the SDG approximation to $\left\langle t^{m}\right\rangle$. We saw that the characteristic mass scale of gluons contributing to $\left\langle t^{m}\right\rangle$ increases fast with $m$ (see table 3). Whereas typical gluon virtuality contributing to the average thrust is about $10 \%$ of the center-of-mass energy $Q$, this fraction becomes $27 \%$ for $<t^{2}>$. Still, if one is using the $\overline{\mathrm{MS}}$ scheme, the natural renormalization scale $\mu_{\mathrm{BLM}, \overline{\mathrm{MS}}}$ is quite far from the naive choice $\mu=Q$ : for $\left\langle t^{2}\right\rangle$ we have $\mu_{\mathrm{BLM}, \overline{\mathrm{MS}}} \simeq 0.12 Q$. This means that the effect of the resummation compared to the naive perturbative treatment is still very significant in the case of $<t^{2}>$.

For high moments $m \geq 3$, a significant source of uncertainty in the available next-to-leading order perturbative approximation (see eq. (3.7)) is related to multijet configurations that contribute at high values of $t, t \gtrsim 1 / 3$. Improving the approximation requires a full next-to-next-to-leading order calculation.

Our renormalon analysis was performed in the inclusive "massive gluon" approach. The advantage of this approach, apart from its simplicity, is that it naturally generalizes to include higher orders [7] in the $\beta$ function of the "skeleton scheme". In addition, it allows for non-perturbative effects to be parametrized [6] in a transparent way using an infrared regular running-coupling. Using this parameterization the universality assumption can be tested. Of course, the inclusive resummation is justified only if the non-inclusive effect is small. An alternative approach to perform renormalon resummation is based on the "naive non-Abelianization" procedure. One can define non-inclusive large $N_{f}$ characteristic functions (see [22, 26]) for $\left\langle t^{m}>\right.$, and then restore the full $\beta_{0}$ of the non-Abelian theory. Comparing the expansion of the inclusive resummation formula (3.1) to the next-to-leading order perturbative expansion, we obtained a quantitative estimate of the non-inclusive effect. The discrepancy at the next-to-leading order is very small (4.4\%) for $<t>$ and it increases for higher moments. In the case of $\left\langle t^{2}>\right.$ the inclusive approximation still seems reasonable (a discrepancy of $18.7 \%$ ) but less so for $<t^{3}>$. Due to the appreciable discrepancy it is worthwhile to compute also the non-inclusive resummation.

We find that within the framework of the SDG calculation, power-corrections to $\left\langle t^{m}>\right.$, with $m \geq 2$, are highly suppressed (see tables 4 and 5). The main question that remains open concerning the phenomenology of $\left\langle t^{2}\right\rangle$ is the significance of power-corrections from configurations of three hard partons plus a soft gluon. Such corrections can be [0, 11, 12, 14] as large as $\alpha_{s}\left(Q^{2}\right) / Q$. We further address this 
subject below.

In the case of the average thrust the leading ambiguity of the perturbative sum scales as $1 / Q$. This ambiguity originates in a particular part of phase-space where a soft gluon is emitted at a large angle. Being associated with large distance physics, it is quite clear that this ambiguity can be resolved only at the non-perturbative level, by including explicit non-perturbative terms that fall as $1 / Q$. We showed that for higher moments of 1 - thrust the infrared contribution to the SDG perturbative sum is dominated by the same large-angle soft-gluon region of phase-space. The corresponding non-analytic terms in the characteristic functions lead to much suppressed power-corrections, which scale as $1 / Q^{3}$ for $<t^{2}>$ and $<t^{3}>$ and as $1 / Q^{5}$ for $<t^{4}>$.

For the various moments of $1-$ thrust, $\left\langle t^{m}\right\rangle$, as for any other time-like observable, there are further ambiguities in the summation of perturbation theory that are not related to large distance scales. Such ambiguities become apparent when comparing different regularizations, such as the principal-value Borel-sum and the APT integral. In the case of the moments of $1-$ thrust, these ambiguities scale as $1 / Q^{2}$ and thus dominate over the infrared ambiguity for $m \geq 2$. We saw that this type of ambiguity is not associated to any particular part of phase-space. This suggest that, contrary to the soft emission ambiguities, it does not signal any genuine non-perturbative effects. It may be then possible to eliminate the ambiguity just by choosing the correct class of regularizations of the perturbative sum. The simplest possibility is that this class is defined by a space-like momentum cutoff [30, 7], or equivalently by the principal-value Borel-sum. One should be aware that other scenarios 31] are possible as well.

Finally, we would like to view our conclusions concerning the power-corrections for the moments of 1 - thrust in the SDG model in the context of previous analysis of the thrust distribution. For this purpose we briefly recall the results of refs. [5, 11, 12. Assuming a two-jet configuration, it was shown that the main effect of nonperturbative soft gluon emission is a shift of the Sudakov-resummed perturbative spectrum to higher values of $t$ :

$$
\frac{d \sigma}{d t}(t)=\left.\frac{d \sigma}{d t}\right|_{\mathrm{PT}}(t-\Delta t),
$$

where $\Delta t=\lambda / Q$. A priori, this formula applies only in the range $\Delta t \ll t \ll 1 / 3$. It was demonstrated [5] that the measured thrust distribution can be fitted over a large range of $t$ and $Q$ values by introducing such a shift.

It is clear that (4.1) cannot describe the physical distribution at extremely small values of $t, t \lesssim \Delta t$, where multiple non-perturbative soft gluon emission becomes essential. This difficulty can be resolved [11, 12], by introducing a non-perturbative (observable dependent) shape-function (SF) to describe the energy flow in the final state. As explained in [12], in this case the physical distribution is obtained by a convolution of the Sudakov-resummed perturbative spectrum with the shape-function. 
The resulting distribution at extremely small $t$ (to the left of the distribution peak) depends on the form of the shape-function but at higher $t$ it approximately coincides with the shifted distribution (4.1).

Both the simple shift model [5] and the shape-function approach [11, 12], like the Sudakov-resummed perturbative spectrum on which they are based, strongly rely on the two-jet kinematics. These approaches strictly do not apply to the large $t$ region where one gluon becomes hard. The success of the fits [5, 11, 12 in a large range of $t$ and $Q$ values is encouraging and it might suggest that also the first few moments of 1 - thrust could be studied in this framework. Yet, one should be aware of the fact that the distribution is rather flat at large $t$ (where one gluon becomes hard) and thus the total effect of the shift, or the convolution with the shape-function, is minor there. On the other hand the first moments crucially depend on this very same region of $t$. Therefore it is dangerous to analyze the non-perturbative corrections to the moments relying on the apparent success of the fits to the distribution.

Nevertheless, it is interesting to see the consequences of the assumption that the simple shift model [5] or the shape-function approach [11, 12] apply beyond the small $t$ region. In this case one finds [11, 12, 13,

$$
\begin{aligned}
<t>_{\mathrm{SF}} & =<t>_{\mathrm{PT}}+\lambda_{1} / Q \\
<t^{2}>_{\mathrm{SF}} & =<t^{2}>_{\mathrm{PT}}+2 \lambda_{1}<t>_{\mathrm{PT}} / Q+\lambda_{2} / Q^{2},
\end{aligned}
$$

where the scales $\lambda_{i}$ are the moments of the shape-function and in the case of a shift (4.1), they are simply $\lambda_{i}=\lambda^{i}$. This can be compared to the infrared powercorrections found in the SDG calculation (table 1), namely

$$
\begin{aligned}
<t>_{\mathrm{SDG}} & =<t>_{\mathrm{PT}}+\lambda / Q+\mathcal{O}\left(1 / Q^{3}\right) \\
<t^{2}>_{\mathrm{SDG}} & =<t^{2}>_{\mathrm{PT}}+\mathcal{O}\left(1 / Q^{3}\right)
\end{aligned}
$$

baring the fact that the perturbative part $\left\langle t^{m}>_{\mathrm{PT}}\right.$ can be quite different in the two approaches: in (4.2) Sudakov resummation is implicitly assumed while in (4.3) SDG renormalon resummation is assumed.

For the average thrust the two approaches predict the same type of leading power-correction. Let us examine the case of $\left\langle t^{2}\right\rangle$ :

(i) the leading non-perturbative term in (4.2), $2 \lambda_{1}<t>_{\mathrm{PT}} / Q$, is proportional to $\alpha_{s}\left(Q^{2}\right)$. Therefore, this term is attributed to soft emission around a configuration of three hard partons. Clearly, this is beyond the scope the SDG calculation performed here. On the other hand, this term crucially depends on the extension of the distribution models of [5, 11, 12] beyond their range of validity (two-jet kinematics). Therefore further theoretical work is required. Note that this prediction for the leading power-correction of $\left\langle t^{2}\right\rangle$ is rather easy to verify, or exclude, using experimental data: due to the large ratio 
(see eq. (3.6)) between the normalization of $\langle t\rangle_{\mathrm{PT}}$ and $\left\langle t^{2}\right\rangle_{\mathrm{PT}}$ in (4.2), the numerical significance of this power-correction is quite large.

(ii) the second term in (4.2),$\lambda_{2} / Q^{2}$, is not suppressed by any power of $\alpha_{s}$, and is therefore attributed to soft emission around the two-jet configuration. On the other hand, the SDG calculation (4.3) does not yield any infrared $1 / Q^{2}$ correction to $<t^{2}>_{\mathrm{PT}}$. There are two possibilities how these facts can be reconciled. The first is that once a more complete ${ }^{\dagger \dagger}$ description of the thrust distribution is achieved there will not be any infrared $1 / Q^{2}$ correction to $<t^{2}>$. The second is that an infrared $1 / Q^{2}$ correction will emerge entirely from double gluon emission. It is a possible, but yet an unusual, situation that double soft gluon emission becomes parametrically less suppressed than a single soft gluon emission around the same hard configuration. Therefore it will be interesting to investigate power-corrections to $<t^{2}>$ from double gluon emission within the renormalon approach.

We comment that techniques for the systematic analysis of power-corrections in the full four parton phase-space are not well established yet. The discussion above clearly shows that such techniques are necessary for the analysis of event-shape variables.

\section{Acknowledgments}

I am grateful to Yuri Dokshitzer, Georges Grunberg and Gregory Korchemsky for very interesting and useful discussions and to Otmar Biebel for his great help.

\footnotetext{
${ }^{\dagger \dagger}$ The shape-function approach resums the power-corrections to the distribution which are the most singular in the small $t$ limit, i.e. corrections that scale as $1 /\left(Q^{n} t^{n}\right)$ for any $n$. Less singular corrections to the distribution, e.g. $1 /\left(Q^{n} t^{n-1}\right)$ can be quite important for the moments.
} 


\section{A. Asymptotic expansions for the characteristic functions}

The asymptotic expansions of $\mathcal{F}_{<t^{m}>}(\epsilon)$, calculated according to eq. (3.8) or (3.9) are the given in eq. (A.1) through (A.4) below. Table 6 summarizes the numerical values of the coefficients.

$$
\begin{aligned}
& \mathcal{F}_{<t>}(\epsilon)=-2 \operatorname{dilog} 3-\frac{1}{6} \pi^{2}-\frac{1}{36}-\frac{3}{8} \ln 3 \\
& -4 \sqrt{\epsilon}+[2+6 \ln 3] \epsilon-\frac{80}{9} \epsilon^{3 / 2} \\
& +\left[\frac{28}{3} \ln 2+2 \operatorname{dilog} 3-\frac{3}{2} \ln \left(\frac{1}{\epsilon}\right)+\frac{17}{12}+\frac{1}{6} \pi^{2}\right] \epsilon^{2}-4 \epsilon^{5 / 2} \\
& +\left[\frac{8}{3} \ln \left(\frac{1}{\epsilon}\right)+\frac{14}{15} \ln 2-\frac{4}{5}\right] \epsilon^{3}+\left[-\frac{64}{105} \ln 2-\frac{11}{2} \ln \left(\frac{1}{\epsilon}\right)+\frac{2719}{1260}\right] \epsilon^{4}+\cdots \\
& \mathcal{F}_{<t^{2}>}(\epsilon)=-2 \operatorname{dilog} 3-\frac{1}{6} \pi^{2}-\frac{9}{8} \ln 3+\frac{17}{216} \\
& +\left[12 \operatorname{dil} \log 3+\pi^{2}-\frac{91}{18}+\frac{127}{12} \ln 3\right] \epsilon+\frac{16}{9} \epsilon^{3 / 2} \\
& +\left[\frac{11}{6} \pi^{2}+\frac{1}{4} \ln \left(\frac{1}{\epsilon}\right)+22 \operatorname{dilog} 3-13 \ln 3+\frac{104}{3} \ln 2+\frac{4}{3}\right] \epsilon^{2}+\frac{16}{9} \epsilon^{5 / 2} \\
& +\left[8 \operatorname{dilog} 3+\frac{188}{15} \ln 2-\frac{1}{3} \ln \left(\frac{1}{\epsilon}\right)-\frac{103}{30}+\frac{2}{3} \pi^{2}\right] \epsilon^{3} \\
& +\left[\frac{5}{12} \ln \left(\frac{1}{\epsilon}\right)-\frac{1003}{315}+\frac{152}{105} \ln 2\right] \epsilon^{4}+\cdots \\
& \mathcal{F}_{<t^{3}>}(\epsilon)=-2 \operatorname{dilog} 3-\frac{1}{6} \pi^{2}+\frac{28}{135}-\frac{83}{64} \ln 3 \\
& +\left[\frac{8}{3} \pi^{2}+\frac{373}{16} \ln 3-\frac{53}{9}+32 \operatorname{dilog} 3\right] \epsilon-\frac{4}{9} \epsilon^{3 / 2} \\
& +\left[\frac{227}{12}+\frac{23}{6} \pi^{2}+76 \ln 2+46 \operatorname{dil} \log 3-\frac{155}{4} \ln 3\right] \epsilon^{2}-\frac{128}{225} \epsilon^{5 / 2} \\
& +\left[-\frac{1127}{180}+\frac{302}{15} \ln 2+\pi^{2}+12 \operatorname{dilog} 3\right] \epsilon^{3}-\frac{4}{9} \epsilon^{7 / 2} \\
& +\left[-\frac{1}{4} \ln \left(\frac{1}{\epsilon}\right)-\frac{34999}{5040}+\frac{1201}{105} \ln 2\right] \epsilon^{4}+\cdots \\
& \mathcal{F}_{<t^{4}>}(\epsilon)=-2 \operatorname{dilog} 3-\frac{1}{6} \pi^{2}+\frac{1259}{4860}-\frac{649}{480} \ln 3 \\
& +\left[-\frac{85}{9}+\frac{3373}{80} \ln 3+5 \pi^{2}+60 \operatorname{dilog} 3\right] \epsilon \\
& +\left[\frac{4879}{108}+\frac{400}{3} \ln 2+\frac{5}{2} \pi^{2}-\frac{326}{3} \ln 3+30 \operatorname{dil} \log 3\right] \epsilon^{2}+\frac{32}{75} \epsilon^{5 / 2} \\
& +\left[-\frac{841}{30}-\frac{824}{15} \ln 2-\frac{16}{3} \pi^{2}+24 \ln 3-64 \operatorname{dilog} 3\right] \epsilon^{3}+\frac{32}{75} \epsilon^{7 / 2} \\
& +\left[-\frac{5849}{315}+\frac{372}{35} \ln 2-8 \ln 3-\frac{8}{3} \pi^{2}-32 \operatorname{dil} \log 3\right] \epsilon^{4}+\cdots
\end{aligned}
$$




\begin{tabular}{|c|c|c|c|c|c|}
\hline term & IR & $\langle t\rangle$ & $\left\langle t^{2}\right\rangle$ & $\left\langle t^{3}\right\rangle$ & $\left\langle t^{4}\right\rangle$ \\
\hline \hline$\epsilon^{0}$ & & +0.7888 & +0.0713 & +0.01120 & +0.002197 \\
\hline$\epsilon^{1 / 2}$ & $1 / Q$ & -4 & & & \\
\hline$\epsilon^{1}$ & & +8.5917 & -0.7999 & +0.06557 & +0.019036 \\
\hline$\epsilon^{3 / 2}$ & $1 / Q^{3}$ & -8.8889 & +1.7778 & -0.4444 & \\
\hline$\epsilon^{2} \ln \left(\frac{1}{\epsilon}\right)$ & $1 / Q^{4}$ & -1.5 & +0.25 & & \\
\hline$\epsilon^{2}$ & & +6.6575 & -2.4337 & +0.76777 & -0.215365 \\
\hline$\epsilon^{5 / 2}$ & $1 / Q^{5}$ & -4 & +1.7778 & -0.56889 & +0.426667 \\
\hline$\epsilon^{3} \ln \left(\frac{1}{\epsilon}\right)$ & $1 / Q^{6}$ & +2.6667 & -0.3333 & & \\
\hline$\epsilon^{3}$ & & -0.1531 & +0.3399 & +0.32290 & -0.429646 \\
\hline$\epsilon^{7 / 2}$ & $1 / Q^{7}$ & & & -0.4444 & +0.426667 \\
\hline
\end{tabular}

Table 6: Numerical values of the coefficients in the asymptotic expansions of the characteristic functions for various moments of $t \equiv 1-T$. For example, $\mathcal{F}_{\langle t\rangle}(\epsilon)=$ $0.7888-4 \sqrt{\epsilon}+8.5917 \epsilon+\cdots$

Substituting only the upper limit $\sqrt{\epsilon}$ in the second integral in eq. (3.9) we can isolate the large-angle soft-gluon contribution to $\mathcal{F}_{<t^{m}>}(\epsilon)$. The results are the following:

$$
\begin{aligned}
\left.\mathcal{F}_{<t>}(\epsilon)\right|_{\text {large-angle }} & =-4 \sqrt{\epsilon}-\epsilon \ln \left(\frac{1}{\epsilon}\right)-\frac{80}{9} \epsilon^{3 / 2}-\epsilon^{2} \ln \left(\frac{1}{\epsilon}\right)-4 \epsilon^{5 / 2} \\
\left.\mathcal{F}_{<t^{2}>}(\epsilon)\right|_{\text {large-angle }} & =-\epsilon+\frac{16}{9} \epsilon^{3 / 2}-\left[\frac{1}{4} \ln \left(\frac{1}{\epsilon}\right)+\frac{9}{4}\right] \epsilon^{2}+\frac{16}{9} \epsilon^{5 / 2}-\epsilon^{3} \\
\left.\mathcal{F}_{<t^{3}>}(\epsilon)\right|_{\text {large-angle }} & =-\frac{4}{9} \epsilon^{3 / 2}+\frac{3}{4} \epsilon^{2}-\frac{128}{225} \epsilon^{5 / 2}+\frac{3}{4} \epsilon^{3}-\frac{4}{9} \epsilon^{7 / 2} \\
\left.\mathcal{F}_{<t^{4}>}(\epsilon)\right|_{\text {large-angle }} & =-\frac{1}{4} \epsilon^{2}+\frac{32}{75} \epsilon^{5 / 2}-\frac{7}{18} \epsilon^{3}+\frac{32}{75} \epsilon^{7 / 2}-\frac{1}{4} \epsilon^{4}
\end{aligned}
$$

Note that (A.5) presents exact results rather than asymptotic expansions at small $\epsilon$. 


\section{References}

[1] Yu.L. Dokshitzer, Perturbative QCD and power-corrections, hep-ph/9911299.

[2] Ch. Berger at al., The Pluto Collaboration, "Energy Dependence of Jets Measures in $e^{+} e^{-}$annihilation", em Z. Phys. C12 (1982) 297.

[3] B. R. Webber, Phys. Lett. B339 (1994) 148, hep-ph/9408222].

[4] Yu.L. Dokshitzer and B.R. Webber, Phys. Lett. B352 (1995) 451.

[5] Yu.L. Dokshitzer and B.R. Webber, Phys. Lett. B404 (1997) 321.

[6] Yu.L. Dokshitzer, G. Marchesini and B.R. Webber, Nucl. Phys. B469 (1996) 93.

[7] E. Gardi and G. Grunberg, JHEP 11 (1999) 016, hep-ph/9908458.

[8] P. Nason and M.H. Seymour, Nucl. Phys. B454 (1995) 291.

[9] R. Akhoury and V. I. Zakharov, Phys. Lett. B357 (1995) 646, hep-ph/9504248].

[10] G.P. Korchemsky and G. Sterman, 30th Recontres de Moriond, QCD and high energy hadronic interactions, Les-Arcs, Savoie, France, 18-25 March, 1995. ed. J. Tran Thanh Van (Editions Frontieres, Gif-sur-Yvette, 1995), p.383, hep-ph/9505391

[11] G. P. Korchemsky, Shape functions and power-corrections to the event shapes, hep$\mathrm{ph} / 9806537$.

[12] G. P. Korchemsky and G. Sterman, Nucl. Phys. B555 (1999) 335.

[13] B.R. Webber, Renormalon phenomena in jets and hard processes, Talk given at 27th International Symposium on Multiparticle Dynamics (ISMD 97), Frascati, Italy, 8-12 Sep 1997. Nucl. Phys. Proc. Suppl. 71 (1999) 66, hep-ph/9712236.

[14] For a recent review see M. Beneke, Renormalons, Phys. Rept. 317 (1999) 1, hep$\mathrm{ph} / 9807443$.

[15] The L3 Collaboration, L3 Note 2414, QCD Studies and Determination of $\alpha_{s}$ using Hadronic Event Structures from $30 \mathrm{GeV}$ to $189 \mathrm{GeV}$, Presented at the International Europhysics Conference High Energy Physics 99, Tampere, Finland, July 1999.

[16] D. Wicke, power-corrections at LEP, hep-ph/9805392. Presented at the 33rd Rencontres de Moriond: Electroweak Interactions and Unified Theories, Les Arcs, France, March 1998.

[17] Private communication from Otmar Biebel.

[18] S. Catani, L. Trentadue, G. Turnock and B.R. Webber, Nucl. Phys. B407 (1993) 3; Phys. Lett. B263 (1991) 491.

[19] R.K. Ellis, D.A. Ross and A.E. Terrano, Nucl. Phys. B178 (1981) 421. 
[20] Z. Kunszt, P. Nason, QCD, in Z physics at LEP 1 vol. 1, G. Altarelli, R. Kleiss, and G. Verzegnassi eds.

[21] S. Catani and M.H. Seymour Phys. Lett. B378 (1996) 287, http://hepwww.rl.ac.uk/theory/seymour/nlo/

[22] M. Beneke, V.M. Braun and L. Magnea, Nucl. Phys. B497 (1997) 297.

[23] G.P. Korchemsky and G. Sterman, Nucl. Phys. B437 (1995) 415.

[24] S. J. Brodsky, E. Gardi, G. Grunberg and J. Rathsman, Conformal expansions and renormalons, hep-ph/0002065.

[25] S.J. Brodsky, G.P. Lepage and P.B. Mackenzie, Phys. Rev. D28 (1983) 228; G.P. Lepage and P.B. Mackenzie, Phys. Rev. D48 (1993) 2250.

[26] M. Dasgupta, L. Magnea and G. Smye, JHEP 11 (1999) 025, [hep-ph/9911316].

[27] G. Altarelli, Phys. Rept. 81 (1982) 1.

[28] S. Kluth, analysis of $N_{c}$ and $N_{f}$ dependence based on a modification of the program EVENT2 21].

[29] M. Beneke and V.M. Braun, Phys. Lett. B348 (1995) 513; P. Ball, M. Beneke and V.M. Braun, Nucl. Phys. B452 (1995) 563.

[30] G. Grunberg, JHEP 11 (1998) 006; hep-ph/9807494.

[31] I.L. Solovtsov and D.V. Shirkov, Phys. Rev. Lett. 79 (1997) 1209.

[32] M. Beneke, V.M. Braun and V.I. Zakharov, Phys. Rev. Lett. 73 (1994) 3058. 